\title{
A controllable double-cycle cryogenic device inducing hypothermia for laparoscopic orthotopic kidney transplantation in swine
}

\author{
Peng Zhang, Xiuwu Han, Xin Zhang, Xuhui Zhu, Tao Li, Yansheng Li, Yuanhao Chen, Gao Li, \\ Longxi Han, Rongjie Zhang \\ Department of Urology, Beijing Chaoyang Hospital, Capital Medical University, Beijing, China \\ Contributions: (I) Conception and design: X Han, P Zhang; (II) Administrative support: X Han, X Zhang, X Zhu; (III) Provision of study materials or \\ patients: T Li, Y Li, Y Chen, G Li; (IV) Collection and assembly of data: L Han, R Zhang; (V) Data analysis and interpretation: P Zhang, X Han; \\ (VI) Manuscript writing: All authors; (VII) Final approval of manuscript: All authors. \\ Correspondence to: Xiuwu Han. Department of Urology, Beijing Chaoyang Hospital, Capital Medical University, Beijing 100043, China. \\ Email: xiuwuhan@163.com.
}

Background: It is necessity to maintain donor kidneys at a low temperature for laparoscopic orthotopic kidney transplantation (LOKT). We recently designed a controllable double-cycle cryogenic device (DCD) with a safer and more effective cooling setting. This study aimed to evaluate the safety and efficacy of this device in a swine model.

Methods: A total of 20 pigs were procured for this study. We performed LOKT on 8 pigs using the controllable DCD for hypothermia, on another 8 pigs using the silicon tube cage (STC) for hypothermia, and on 4 pigs as the control group with no hypothermia measures (NH). The LOKTs were performed in autotransplantation mode in which the right kidney vein and artery were end-to-end anastomosed to the left kidney vein and artery in all three groups. The perioperative data, graft temperatures, and surgical complications were recorded, and histological examinations of kidney specimens were performed.

Results: The average surface temperature in the DCD group $\left(5 \pm 3.5^{\circ} \mathrm{C}\right)$ was significantly lower than that in the STC $\left(10 \pm 5.5^{\circ} \mathrm{C}\right)$ and $\mathrm{NH}\left(28 \pm 3.0^{\circ} \mathrm{C}\right)$ groups. In the DCD and STC groups, the temperature between the cooling setting and the adjacent peritoneum was $30 \pm 3.0$ and $12 \pm 5.0^{\circ} \mathrm{C}$, respectively. All pigs survived for 7 days in the DCD group. In the STC group, 2 pigs died from liver lesions and other causes. A single pig had diabetes insipidus. No pigs survived for 7 days in the NH group. The specimens from pigs immediately after revascularization and from the surviving pigs on day 7 following transplantation showed that the morphology of the glomerular and renal tubules was largely normal in the DCD and STC groups. However, in the NH group, there was minimal interstitial and tubular oedema, and endothelial cell swelling occurred in some specimens immediately after revascularization. Pathological examination of the harvested graft specimens showed severe acute tubular necrosis in the NH group.

Conclusions: The newly designed controllable DCD can achieve the goal of cooling the kidney and warming the patient and may be a reliable, effective, inexpensive, and practical hypothermic maintenance system for LOKT in swine.

Keywords: Laparoscopy; hypothermia; device; kidney transplantation; orthotopic

Submitted Jun 04, 2021. Accepted for publication Jul 14, 2021.

doi: $10.21037 /$ tau-21-544

View this article at: https://dx.doi.org/10.21037/tau-21-544 


\section{Introduction}

In recent years, laparoscopy or robot-assisted laparoscopy has been employed in kidney and pancreas transplantation, demonstrating its inherent advantages of reduced trauma, faster recovery, lower infection rate, and equal or improved clinical outcomes (1-5). Laparoscopic kidney transplantation requires not only skilled vascular anastomosis but also reliable methods to maintain organs at a low temperature during vascular anastomosis. For surgeons who perform clinical laparoscopic kidney transplantation, it is necessary to maintain the donor kidney at a low temperature during vascular anastomosis. If the vascular anastomosis takes too long for various reasons, there are no reliable procedures to induce hypothermia, which may lead to adverse consequences $(6,7)$. On the other hand, excessive hypothermia of the operation field caused by the cooling method is also a large risk factor for patients, especially in laparoscopic orthotopic kidney transplantation (LOKT). Therefore, a reliable intracorporeal cooling device should be researched and developed. In recent years, we have conducted experimental research on LOKT and designed cooling equipment for laparoscopic organ transplantation $(8,9)$. Based on previous designs, after refinement and improvement, we recently designed a safer and more effective cooling setting, a controllable double-cycle cryogenic device (DCD; patented in China, patent No. ZL2018 2 0687164.X), to prevent rewarming during LOKT. This study evaluated the safety and efficacy of this device in a preclinical swine model. We present the following article in accordance with the ARRIVE reporting checklist (available at https://dx.doi.org/10.21037/ tau-21-544).

\section{Methods}

\section{Animals and preparation}

A total of 20 swine provided by Beijing Tonghe Litai Biotechnology Co., Ltd. were available for a LOKT trial to assess the safety and reliability of the organ cooling system. The animals were divided into three groups: 8 in the cooling jacket group using the controllable DCD for hypothermia (DCD group), 8 in the silicon tube cage [STC; as we previously described a device for $\operatorname{LOKT}(8,9)$ ] group using the STC for hypothermia (STC group) and four in the control group involving no hypothermia measures $(\mathrm{NH}$ group).

The swine, weighing $45-50 \mathrm{~kg}$, were transported to the animal experimental laboratory 2 days before the experiment. All pigs were fasted for $12 \mathrm{~h}$ before surgery. The animal experiments were performed under a project license (No. 2014-Ke-136) granted by the research committee and the institutional animal care and use committee of Beijing Chaoyang Hospital. A protocol was prepared before the study without registration.

\section{Cooling device preparation}

\section{Controllable DCD}

First, an airtight plastic bag, approximately $12 \mathrm{~cm}$ wide and $20 \mathrm{~cm}$ long, was constructed, with two silicone or latex tubes, and an end depth less than half of the long side (approximately 4-8 cm) on both of the long sides of the bag (Figure 1A). When in use, the bottom of the bag (the opposite side of the catheter input) was invaginated (Figure 1B), the kidney was encapsulated, and the mouth formed after the invagination was closed with titanium clips or silk thread ligation multiple times at several sites to wrap the donor kidney. Only an approximately $3.0 \mathrm{~cm}$ hole was left open at the renal hilum for renal artery and vein exposure. A silicone tube pad with two long tubular tails, approximately $8-10 \mathrm{~cm}$ in diameter, was made by coiling and fixing with silk thread, from which the two ends of the silica gel tube could be used for warm water inflow and outflow. It was placed immediately behind the plastic bag in the ventral position of the donor kidney facing the pelvic viscera to protect the ventral organs from the cold during the operation (Figure 1A,C). Finally, the plastic bag containing the donor kidney and the silicone tube pad were wrapped in a net bag to fix the pad in position and limit excessive bag swelling. At the hilum of the kidney, corresponding to the hole of the plastic bag, a hole of similar size in the net bag was made to allow simple exposure of the renal artery and vein. With this net, a confined, double-layer plastic bag jacket with a bicirculating system was formed (Figure 1D).

\section{STC}

A previously introduced STC was used in the study. It was formed with the silicon tubes. The silicon tube was extended spirally to approximately $10-12 \mathrm{~cm}$ in length with a diameter of approximately $6-8 \mathrm{~cm}$, and it was then fixed by $4-5$ parallel threads to make a cage-like device. The kidney was placed into the device, and blood vessels extended the outside at the center of the cage. During the operation, ice water was circulated through the silicon tube to maintain hypothermia (Figure 2). 

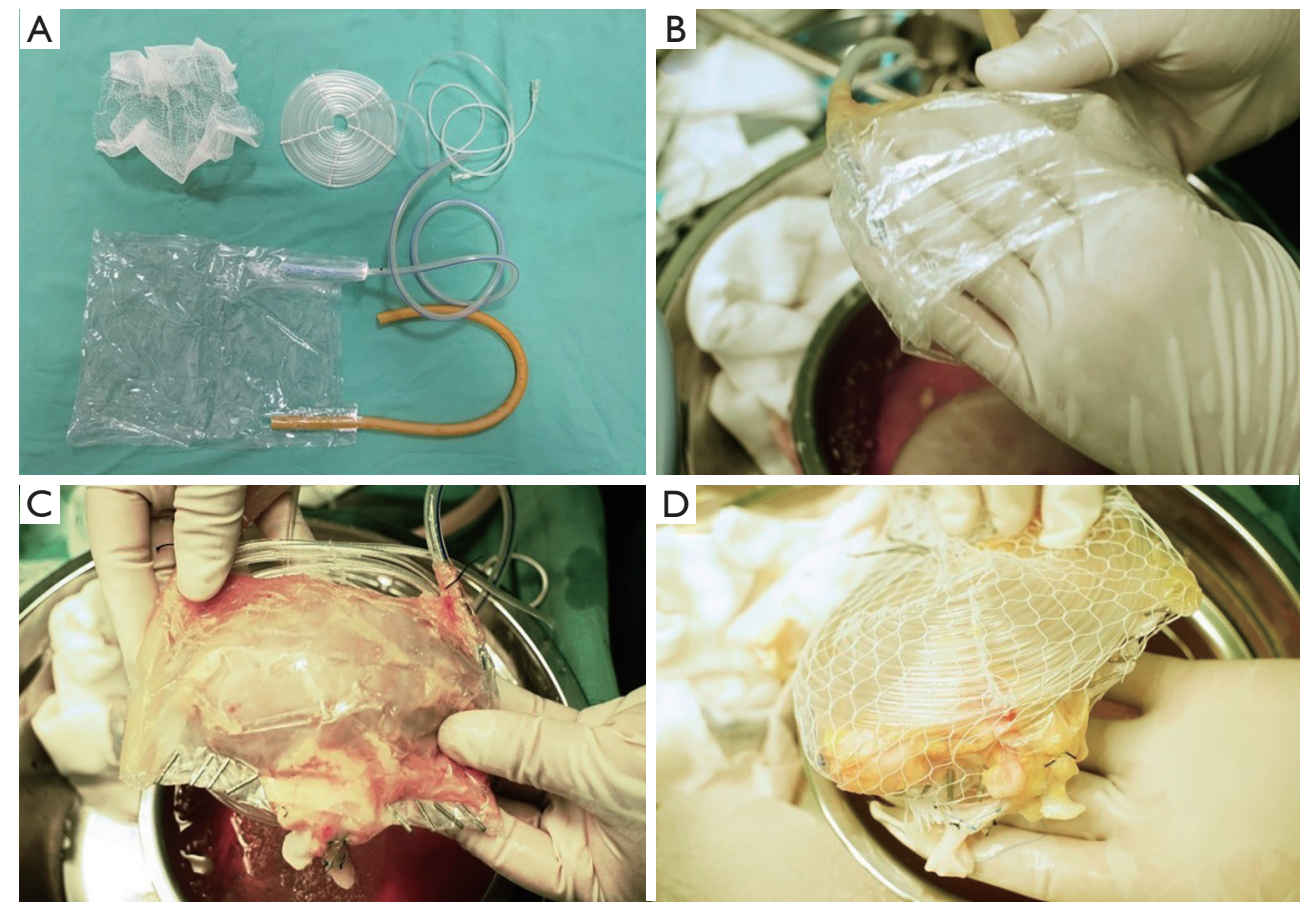

Figure 1 Formation of the net-restrictive bicirculating double-layer plastic bag jacket. (A) Composition and pre combination state of cryogenic device; (B) the cooling bag is folded for kidney transplantation; (C) the graft was placed in the plastic bag and the edge was closed with a titanium clip; (D) all devices are assembled and ready for transplantation.

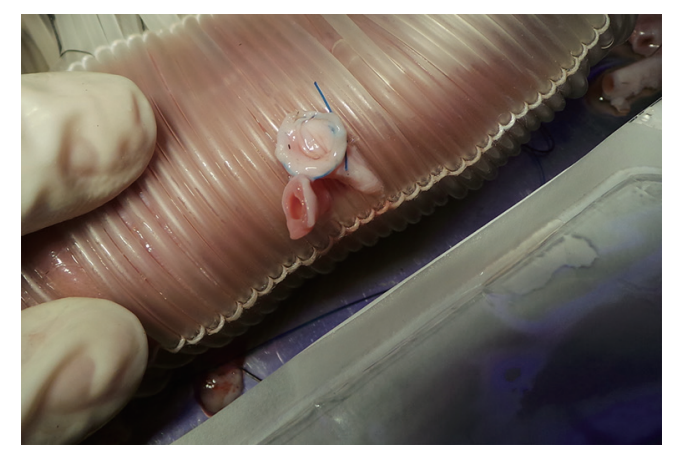

Figure 2 Formation of the STC. STC, silicon tube cage.

\section{Surgery for LOKT}

\section{Nephrectomy}

The LOKTs were conducted in autotransplantation mode in which the right kidney vein and artery were end-to-end anastomosed to the left kidney vein and artery in all three groups. After the induction of general anesthesia in a supine position, a $12-15 \mathrm{~cm}$ incision was made along the midline of the abdomen above the umbilicus. Left and right posterior peritoneal cavities were created, and the left and right kidneys were separated by open procedures. During the operation, the left kidney was selected as the donor kidney and removed first, and preparation for the donor graft was performed. The left kidney vessels were cut as close as possible to the aorta and inferior vena cava, and the ureter was severed at the segment approaching the bladder. The longer renal vessels and ureter were retained along with the kidney, and their ends were firmly ligated. On the right side of the nondonor kidney nephrectomy, small bulldog or titanium clips were used to block the renal arteries and veins, respectively, as close as possible to the aorta and inferior vena cava, and the renal vessels were dissected as close as possible to the renal sinus, leaving the renal artery and vein ends as long as possible for anastomosis during kidney transplantation. Immediately after the donor kidney was removed, lavage was completed with $4{ }^{\circ} \mathrm{C}$ perfusion fluids. Then, the renal vein was belted, meaning that the dissected edge of the renal vein was sutured circumferentially with a 5-0 Prolene thread, and a knot was tied to maintain the renal vein at the maximum diameter, as previously reported (9). The donor kidney was then placed in the cooling device (or not, according to the group) and 


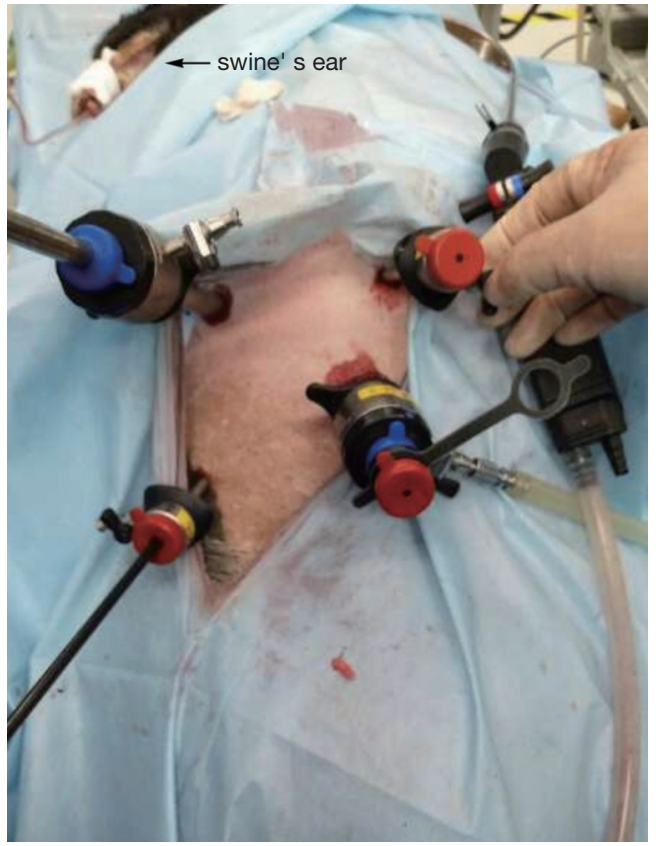

Figure 3 Trocar locations for LOKT in a sample pig. LOKT, laparoscopic orthotopic kidney transplantation.

refrigerated in ice water for later use.

\section{Laparoscopic orthotopic kidney autotransplantation}

The pig was moved to the left lateral position for implanting of the left kidney to the right kidney position after nephrectomy. Normally, four trocars are placed on the right side of the abdominal wall with the original incision under manual guidance, similar to previous reports $(8,9)$. Specifically, the first trocar, which was $5.5 \mathrm{~mm}$, was located $2 \mathrm{~cm}$ above the junction between the iliac crest and the midaxillary line. A second trocar, which was $10.5 \mathrm{~mm}$, was located 6-8 cm above the first and slightly ventrally. A third trocar, which was $10.5 \mathrm{~mm}$, was located ventrally $10-12 \mathrm{~mm}$ from the first, and a fourth trocar, which was $5.5 \mathrm{~mm}$, was 5-6 $\mathrm{cm}$ above the third (Figure 3).

With two strips of thread (No. 10) and a large needle, we continued suturing from both ends of the wound with a large interval to the middle. The threads were long enough to loosen. The donor kidney, together with the cooling device, was placed through the incision, and the donor vessel was placed as close as possible to the renal vessel end. Then, the loosened suture was tightened, and the incision was temporarily closed. Inflows and outflows of circulating cold and warm water ensued. The pneumoperitoneum was started, while $0-4{ }^{\circ} \mathrm{C}$ saline was injected continuously at a speed of $200 \mathrm{~mL} / \mathrm{min}$ by the assistants for the DCD and STC groups, and $30-35{ }^{\circ} \mathrm{C}$ water was instilled continuously at a speed of $100 \mathrm{~mL} / \mathrm{min}$ by a 1.0 meter high infusion set from in the DCD group. The ice water flow was maintained until revascularization. The temperatures were monitored during the operating period using a thermometer (Thermal Detector, DT-613, Shenzhen, China) on both sides of the donor kidney, at the pelvis of the donor kidney, and between the cooling device and the peritoneum (in the NH group, there was no need for the fourth thermal probe between the cooling device and peritoneum). The laterality of the donor kidney, surgical approach, vascular anastomosis step, wound location and size, and so on were all the same in the three groups, and only the organ cooling mode was different.

Anastomoses of the donor renal vessel ends to the recipient renal vessel ends were performed laparoscopically with 5-0 Prolene sutures. After the anastomotic site was checked to ensure that it was free from leakage, the circulation of the device was stopped, and then the blood supply was opened (Figure 4). The incision suture was loosened, and the incision was opened. The cooling device and/or thermal probe was cut and removed from the wound, and then the ureter was placed into a single $\mathrm{J}$ tube and implanted in the vicinity of the skin. Finally, the incision and trocar sites were closed with a suture.

\section{Postoperative care of the animals}

After the operation, intravenous supplement of 1.0-1.5 L $0.9 \%$ sodium chloride solution was administered to the pigs for 2-3 days. The pigs were allowed water ad libitum. After the first postoperative day, the standard diet was offered to pigs. A subcutaneous injection of heparin once a day (3,000 IU) was administered for antithrombotic therapy. Intramuscular ampicillin injection of $500 \mathrm{mg}$ daily was administered for anti-infection treatment. On postoperative day 7 , under general anesthesia, the transplanted kidney was removed, and then anesthetic euthanasia of the pigs was performed. The postoperative serum creatinine, glutamic-pyruvic transaminase, albumin and urine output, pig survival rate, and surgical complications were recorded, and histological examinations of kidney specimens were performed.

Needle biopsies of kidney grafts were taken at 2 time points: immediately after revascularization and on day 7 following transplantation or at the time pigs were examined upon death under general anesthesia. Some tissue samples were immersed in $4 \%$ buffered formalin, and some were fixed in $1 \%$ osmium tetroxide and embedded with 

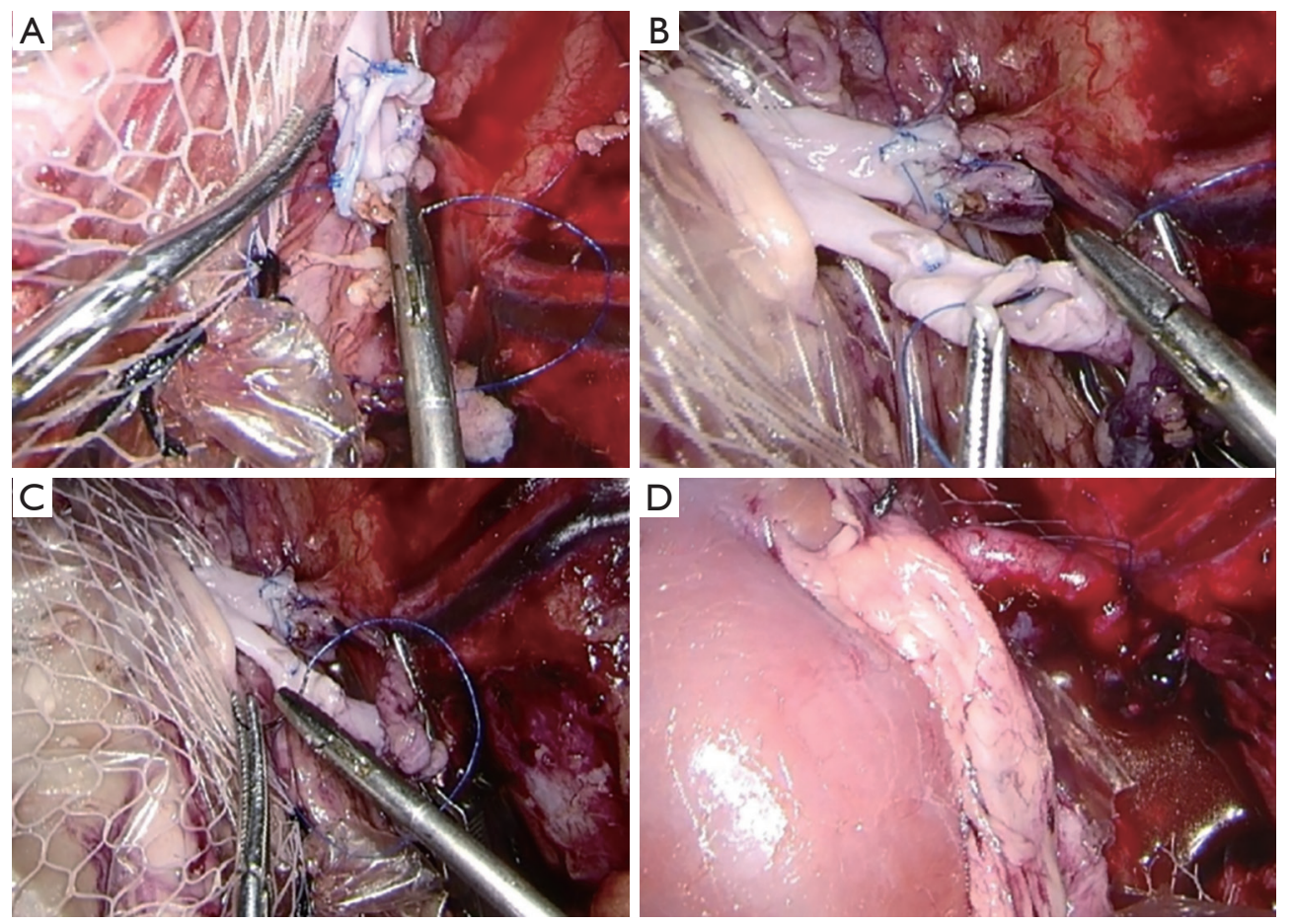

Figure 4 Cooling device in a LOKT in a sample pig. (A) Venous anastomosis of the renal vein with "belting"; (B) arterial anastomosis; (C) the congealed water beads shown in the cooling system bag; (D) a pink-colored appearance after revascularization is shown in a transplanted kidney. LOKT, laparoscopic orthotopic kidney transplantation.

SPI-Pon 812 Embedding Kit (SPI Supplies, USA) for light microscopy and electron microscopy inspection, respectively. Ultrathin sections of the samples were stained with uranyl acetate and lead citrate and then examined under an electron microscope (JEM-1400Plus; Jeol Ltd., Tokyo, Japan).

\section{Statistical analysis}

The results were presented as the mean value \pm standard deviation (SD). Student's $t$-test or a Mann-Whitney U test and one-way analysis of variance (ANOVA) with Bonferroni's multiple test corrections were used to analyze the differences between groups. The software SPSS (version 17.0, IBM Corp., Chicago, IL, USA) was used for statistical calculations. A P value $<0.05$ was considered statistically significant.

\section{Results}

\section{Surgical results and observation indexes}

All cases of laparoscopic orthotopic autotransplantation in the three groups were successfully completed. The total mean operation times in the DCD, STC, and NH groups were $295 \pm 43,317 \pm 40$, and $343 \pm 43 \mathrm{~min}$, respectively. There was no significant difference between the three groups in operative duration. For the DCD and STC groups, placing the donor kidney and cooling settings in the appropriate position required approximately $15-32 \mathrm{~min}$, whereas it took approximately 5-8 min for the $\mathrm{NH}$ group. However, the location of the donor kidney in the NH group easily shifted; hence, the operation time was not shorter than that of the other two groups, and the intraoperative instruments easily caused damage to the donor kidney. There was no significant difference between the warm ischemia time and the cold ischemia time between the three groups. The average surface temperature of the peritoneum side in the DCD, STC, and NH groups was $5 \pm 3.5,10 \pm 5.5$, and $28 \pm 3.0^{\circ} \mathrm{C}$, respectively. The average body surface temperature in the DCD group was significantly lower than that in the STC and NH groups. It is worth noting that in the DCD group and STC group, the temperature between the cooling setting and the adjacent peritoneum was $30 \pm 3.0$ and $12 \pm 5.0{ }^{\circ} \mathrm{C}$, respectively. Significant differences in renal pelvis temperature between groups were not observed until 
Table 1 Perioperative data and outcomes for the groups

\begin{tabular}{|c|c|c|c|c|c|}
\hline Variables & DCD group & STC group & $\mathrm{NH}$ group & $P$ value (DCD vs. STC) & $\mathrm{P}$ value (DCD vs. $\mathrm{NH})$ \\
\hline Operation time (min) & $295.35 \pm 43.5$ & $317.44 \pm 40$ & $343.35 \pm 43$ & 0.987 & 0.886 \\
\hline Warm ischaemia time (min) & $4.0 \pm 0.5$ & $4.5 \pm 0.5$ & $4.5 \pm 0.8$ & 0.230 & 0.223 \\
\hline Cold ischaemia time $(\mathrm{h})$ & $225 \pm 19$ & $205 \pm 30$ & $275 \pm 38$ & 0.879 & 0.765 \\
\hline Artery anastomotic time (min) & $22 \pm 15$ & $26 \pm 12$ & $27 \pm 15$ & 0.442 & 0.515 \\
\hline \multicolumn{6}{|l|}{ Surface temperature of graft $\left({ }^{\circ} \mathrm{C}\right)$} \\
\hline Upside & $5.6 \pm 2.0$ & $9.8 \pm 1.6$ & $27 \pm 8.0$ & 0.000 & 0.000 \\
\hline Peritoneum side & $5 \pm 3.5$ & $10 \pm 5.5$ & $28 \pm 3.0$ & 0.004 & 0.001 \\
\hline Artery stenosis (n) & 0 & 1 & 0 & & \\
\hline Vein stenosis $(n)$ & 0 & 2 & 0 & & \\
\hline Liver lesion (n) & 0 & 2 & 0 & & \\
\hline Diabetes insipidus (n) & 0 & 1 & 0 & & \\
\hline Urine output at day $7(\mathrm{~mL})$ & $1,406 \pm 110$ & $980 \pm 215$ & - & 0.078 & - \\
\hline Scr at day $7(\mu \mathrm{mol} / \mathrm{dL})$ & $290 \pm 60$ & $310 \pm 21$ & - & 0.092 & - \\
\hline BUN at day 7 (mg/dL) & $18.8 \pm 4.2$ & $23.5 \pm 3.3$ & - & 0.051 & - \\
\hline ATN (n) & 0 & 1 & 4 & & \\
\hline
\end{tabular}

DCD, double-cycle cryogenic device; STC, silicon tube cage; $\mathrm{NH}$, no hypothermia measures; Scr, serum creatinine; BUN, blood urea nitrogen; ATN, acute tubular necrosis.

15-25 min after the graft was placed in the surgical field. The mean renal pelvis temperature in the DCD group was $5.5 \pm 3.3^{\circ} \mathrm{C}$, which was significantly lower than the mean renal pelvis temperature in the STC group $\left(9.5 \pm 3.5^{\circ} \mathrm{C}\right)$ and $\mathrm{NH}$ group $\left(22 \pm 4.0^{\circ} \mathrm{C}\right)$, both $\mathrm{P}<0.05$.

\section{Evaluation of postoperative effect}

A total of 7 grafts showed diuresis in the DCD group, 5 grafts showed diuresis in the STC group on the first day after operation, and none showed diuresis in the $\mathrm{NH}$ group. In the DCD group, all pigs survived for 7 days and 1 pig experienced venous thrombosis with serum creatinine values of $1,055 \mathrm{~mol} / \mathrm{L}$ at postoperative day 7 . In the STC group, 1 pig died on postoperative day 4 from autopsyconfirmed venous thrombosis. A single pig died 5.5 days after the operation from renal vein anastomotic stenosis with a liver lesion. Another pig nearly died 7 days after the operation with a liver lesion. A single pig had diabetes insipidus for 6 days after the operation but had good renal function at the end of the experiment. In the $\mathrm{NH}$ group, no pigs survived for 7 days, even though the renal artery and venous anastomosis were patent in 3 pigs, as shown by surgical exploration under general anesthesia (Table 1).

\section{Histopathological results}

In the DCD and STC groups, most of the specimens from immediately after revascularization and on day 7 following transplantation in the surviving pigs showed that the morphology of the glomerular and renal tubules was basically normal (Figure $5 A, B, C, D$ ). However, in the $\mathrm{NH}$ 

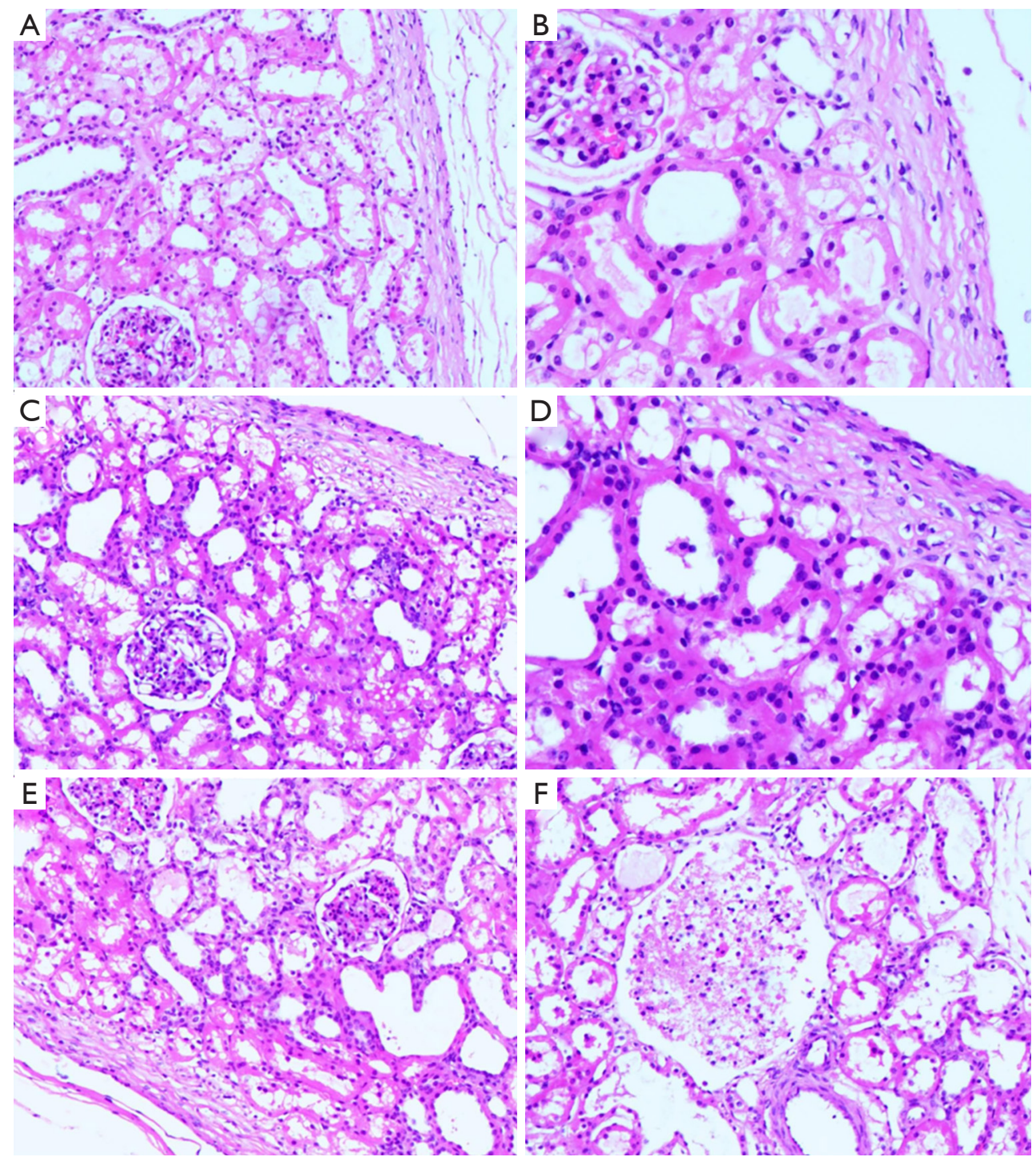

Figure 5 Pathological examination of kidney grafts needle biopsies (hematoxylin-eosin staining). One time point: immediately after revascularization [(A) DCD group; (C) STC group; (E) NH group; 100x]. The other time point: on day 7 following transplantation or at the time of examination upon death under general anesthesia [(B) DCD group; (D) STC group; (F) NH group; 200× ]. DCD, double-cycle cryogenic device; STC, silicon tube cage; NH, no hypothermia measures.

group, there was minimal interstitial and tubular oedema, there was endothelial cell swelling in some specimens immediately after revascularization (Figure $5 E$ ), and pathological examination of the removed graft specimens at the time of examination upon death under general anesthesia showed severe acute tubular necrosis throughout the group (Figure 5F). Under electron microscopy, the glomerular capillary endothelial cells and podocytes were morphologically normal, and the ultrastructure of the renal tubular epithelial cells was nearly normal, with dense and ordered microvilli (Figure $6 A, B, C, D$ ). The specimens from dead or nearly dead pigs showed that the ultrastructure of the glomerular capillary endothelial cells and podocytes were severely destroyed, and the renal tubules had cellular debris, brush border loss, and cellular dilation (Figure 6E,F).

\section{Discussion}

Experimental studies have shown that after cold storage, the metabolic activity in the kidneys resumes at approximately 

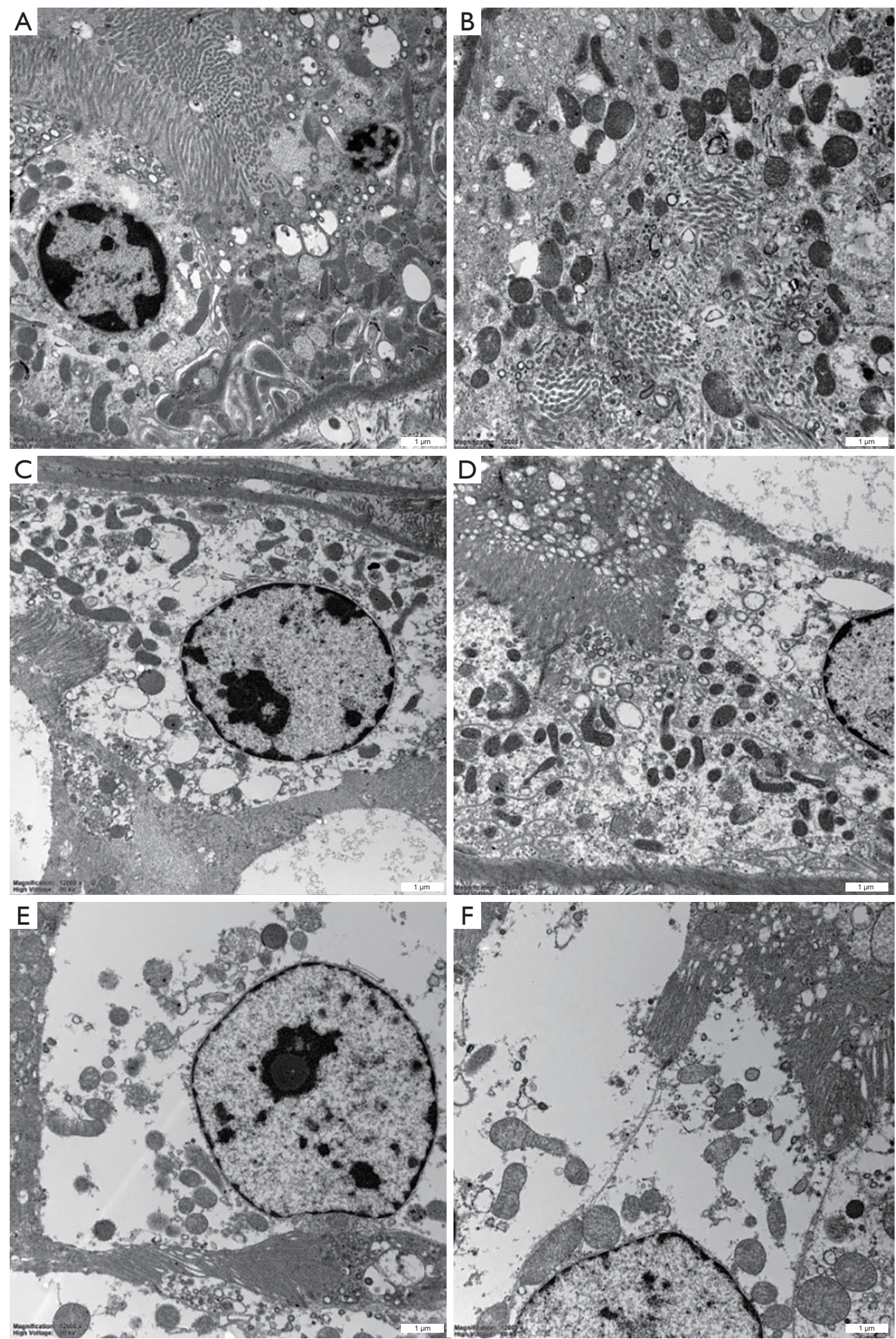

Figure 6 Transmission electron microscopy examination of kidney grafts needle biopsies. One time point: immediately after revascularization [(A) DCD group; (C) STC group; (E) NH group] $(\times 100)$. The other time point: on day 7 following transplantation or at the time of examination upon death under general anesthesia [(B) DCD group; (D) STC group; (F) NH group] ( $\times 200)$. DCD, double-cycle cryogenic device; STC, silicon tube cage; $\mathrm{NH}$, no hypothermia measures. 
$15-18{ }^{\circ} \mathrm{C}(6)$. Another study showed that the renal temperature will rapidly increase to a $15{ }^{\circ} \mathrm{C}$ threshold within $20 \mathrm{~min}$ in more than $80 \%$ of patients during vascular anastomosis in kidney transplantation (10). Therefore, it is very important to preserve the donor kidney in cold storage and maintain a low temperature during the operation to reduce ischemia reperfusion injury and protect the viability of the renal tissue. For laparoscopic or robotassisted laparoscopic kidney transplantation, partial regional hypothermia has been reported to prevent rewarming of the kidney during transplantation. Partial regional hypothermia is achieved by repetitively delivering ice slush into the peritoneum to cover the graft (11-14), by the application of 'jackets' of ice slush to wrap the graft $(6,15)$, a gauzewrapped ice slush placed around the graft (16), simple frequent ice-saline irrigation through the incision (17), or with a standard irrigator/aspirator (18). For the aforementioned techniques used in the operation, surgeons must suction the melted saline repeatedly, which may cause the space to collapse, block their field of vision, and prolong the anastomotic time. A laparoscopic plastic bag with crushed ice has been safely used in robot-assisted kidney transplantation $(6,19)$, but the hypothesized maintenance time may be limited. Meie et al. introduced a commendable intra-abdominal cooling system for robot-assisted renal transplantation in large white pigs with fine results (20). However, these techniques for inducing hypothermia have raised the concern that a patient's core body temperature may be affected $(21,22)$. For LOKT, the cooling site moves upward and is close to the liver, spleen, pancreas, intestines, and other organs, especially the abdominal aorta and inferior vena cava, and core hypothermia is more likely to occur and be more serious. The study results showed liver lesions in 2 pigs and diabetes insipidus in 1 pig in the STC group, which may have been caused by core hypothermia. Therefore, core hypothermia during LOKT surgery must also be addressed and prevented. This study showed that both the DCD and STC can achieve effective low temperature. However, the DCD can not only maintain the cold temperature of the grafts but also protect the receptors.

The new cooling system had the following advantages: (I) it was watertight and airtight, and a reliable cooling effect was achieved; (II) the cooling could be localized to avoid adverse reactions caused by the coldness of the local organs and to achieve the goal of cooling the kidney and warming the patient; (III) the mesh could be used to fix and adjust the position of the kidney for operation; (IV) it protected the kidneys from injury by surgical instrument; and (V) it could wrap the graft geographically, rationally exposing the hilus and occupying a smaller space.

The potential shortcomings of this study were the limited sample size of pigs in the study and the limitation of the observation time. It would be better if a mechanical pump were used for circulation perfusion and if a commercial product version of the device were available.

In conclusion, the controllable DCD may be a reliable, effective, inexpensive, and practical hypothermic maintenance system for LOKT in swine.

\section{Acknowledgments}

Funding: None.

\section{Footnote}

Reporting Checklist: The authors have completed the ARRIVE reporting checklist. Available at https://dx.doi. org/10.21037/tau-21-544

Data Sharing Statement: Available at https://dx.doi. org/10.21037/tau-21-544

Conflicts of Interest: All authors have completed the ICMJE uniform disclosure form (available at https://dx.doi. org/10.21037/tau-21-544). The authors have no conflicts of interest to declare.

Ethical Statement: The authors are accountable for all aspects of the work in ensuring that questions related to the accuracy or integrity of any part of the work are appropriately investigated and resolved. A protocol was prepared before the study without registration. The animal experiments were performed under a project license (No. 2014-Ke-136) granted by the research committee and the institutional animal care and use committee of Beijing Chaoyang Hospital.

Open Access Statement: This is an Open Access article distributed in accordance with the Creative Commons Attribution-NonCommercial-NoDerivs 4.0 International License (CC BY-NC-ND 4.0), which permits the noncommercial replication and distribution of the article with the strict proviso that no changes or edits are made and the original work is properly cited (including links to both the formal publication through the relevant DOI and the license). See: https://creativecommons.org/licenses/by-nc-nd/4.0/. 


\section{References}

1. Rosales A, Salvador JT, Urdaneta G, et al. Laparoscopic kidney transplantation. Eur Urol 2010;57:164-7.

2. Modi P, Rizvi J, Pal B, et al. Laparoscopic kidney transplantation: an initial experience. Am J Transplant 2011;11:1320-4.

3. Modi P, Pal B, Modi J, et al. Retroperitoneoscopic living-donor nephrectomy and laparoscopic kidney transplantation: experience of initial 72 cases. Transplantation 2013;95:100-5.

4. Modi P, Pal B, Kumar S, et al. Laparoscopic transplantation following transvaginal insertion of the kidney: description of technique and outcome. Am J Transplant 2015;15:1915-22.

5. Han X, Zhao Y, Zhang X, et al. Laparoscopic kidney transplantation: a case report. Shandong Medical Journal 2018;58:63-5.

6. Hameed AM, Yuen L, Pang T, et al. Techniques to ameliorate the impact of second warm ischemic time on kidney transplantation outcomes. Transplant Proc 2018;50:3144-51.

7. Boggi U, Signori S, Vistoli F, et al. Current perspectives on laparoscopic robot-assisted pancreas and pancreaskidney transplantation. Rev Diabet Stud 2011;8:28-34.

8. Han X, Zhang B, Yan W, et al. Comparison of 2 devices in pigs to induce hypothermia in laparoscopic orthotopic kidney transplant. Exp Clin Transplant 2012;10:573-8.

9. Han X, Zhao Y, Zhu X, et al. Vein "Beltization" technique facilitates venous anastomoses for laparoscopic orthotopic kidney transplantation in a pig model. Transplant Proc 2019;51:913-8.

10. Kuipers TG, Hellegering J, El Moumni M, et al. Kidney temperature course during living organ procurement and transplantation. Transpl Int 2017;30:162-9.

11. Menon M, Sood A, Bhandari M, et al. Robotic kidney transplantation with regional hypothermia: a step-by-step description of the Vattikuti Urology Institute-Medanta technique (IDEAL phase 2a). Eur Urol 2014;65:991-1000.

12. Abaza R, Ghani KR, Sood A, et al. Robotic kidney transplantation with intraoperative regional hypothermia.

Cite this article as: Zhang $\mathrm{P}$, Han $\mathrm{X}$, Zhang $\mathrm{X}$, Zhu X, Li T, Li Y, Chen Y, Li G, Han L, Zhang R. A controllable doublecycle cryogenic device inducing hypothermia for laparoscopic orthotopic kidney transplantation in swine. Transl Androl Urol 2021;10(7):3046-3055. doi: 10.21037/tau-21-544
BJU Int 2014;113:679-81.

13. Sood A, Ghosh P, Jeong W, et al. Minimally invasive kidney transplantation: perioperative considerations and key 6-month outcomes. Transplantation 2015;99:316-23.

14. Woodworth TG, Furst DE. Timely renal transplantation for scleroderma end-stage kidney disease patients can improve outcomes and quality of life. Ann Transl Med 2019;7:60.

15. Shoskes D. Renal transplantation in urology. Transl Androl Urol 2019;8:108.

16. Peri L, Vilaseca A, Serapiao R, et al. Development of a pig model for laparoscopic kidney transplant. Exp Clin Transplant 2016;14:22-6.

17. Tsai MK, Lee CY, Yang CY, et al. Robot-assisted renal transplantation in the retroperitoneum. Transpl Int 2014;27:452-7.

18. Weld KJ, Koziol S, Montiglio C, et al. Feasibility of laparoscopic renal cooling with near-freezing saline irrigation delivered with a standard irrigator aspirator. Urology 2007;69:465-8.

19. Michiels C, Rouffilange J, Comat V, et al. Total preperitoneal robot-assisted kidney transplantation. J Endourol Case Rep 2017;3:169-72.

20. Meier RPH, Piller V, Hagen ME, et al. Intra-abdominal cooling system limits ischemia-reperfusion injury during robotassisted renal transplantation. Am J Transplant 2018;18:53-62.

21. Ahlawat R, Balakrishnan G, Kher V, et al. Reply to Jyotirmoy Das, Sudhir Kumar, Sangeeta Khanna, and Yatin Mehta's letter to the editor re: Mani Menon, Akshay Sood, Mahendra Bhandari, et al. Robotic kidney transplantation with regional hypothermia: a step-by-step description of the Vattikuti Urology Institute-Medanta Technique (IDEAL Phase 2a). Eur Urol 2014;65:991-1000: robotic kidney transplantation: cool the kidney, warm the patient. Eur Urol 2014;65:e88.

22. Modi P, Pal BC, Kumar S, et al. Outcome of robotic assisted laparoscopic kidney transplantation with and without regional hypothermia. Transplant 2018;102:S346-7.

(English Language Editor: J. Jones) 